\title{
POLÍTICA AGRARIA Y ESTRATEGIA DE DESARROLLO CUBANAS. RETOS PARA EL COOPERATIVISMO.
}

\author{
POLÍTICA AGRÁRIA E ESTRATÉGIA DE DESENVOLVIMENTO CUBANAS. \\ DESAFIOS DO COOPERATIVISMO.
}

\section{AGRARIAN POLICY AND CUBAN DEVELOPMENT STRATEGY. CHALLENGES FOR COOPERATIVISM.}

\author{
Lienny García Pedraza ${ }^{1}$ \\ Jaime García Ruiz ${ }^{2}$ \\ Grizel Donéstevez Sánchez ${ }^{3}$
}

Licença CC BY: Artigo distribuído sob os termos Creative Commons, permite uso e distribuição irrestrita em qualquer meio desde que o autor credite a fonte original.

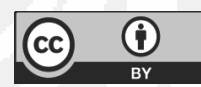

\begin{abstract}
Resumen: El cooperativismo en Cuba se vio potenciado a partir del triunfo de la Revolución del 1 de enero de 1959. A pesar de que el país adoptó la vía de la estatalización como vía de socialización socialista. Apostó por la unidad y cooperación del movimiento campesino cubano para la producción y la prestación de servicios. Por tanto, el problema agrario fue siempre parte de la estrategia de desarrollo de Cuba. Los años recorridos por el cooperativismo agrario y la ampliación del cooperativismo a nuevas esferas de la producción y los servicios; complejizan el escenario actual y plantea nuevos retos. Esto último, pone a la luz la necesidad de mirar el devenir del cooperativismo en Cuba y las políticas que han favorecido o dificultado su desarrollo; sin olvidar la importancia de la participación de los sujetos en la formulación de las mismas. Por ese motivo el presente trabajo pretende caracterizar las acciones del Estado cubano en la potenciación del cooperativismo e identificar sus principales retos en materia de Políticas Públicas. Lo anterior fue posible a partir de una revisión bibliográfica amplia y un análisis de documentos (Leyes, Decretos) que permiten validar el análisis bibliográfico realizado e identificar los retos para el cooperativismo en el nuevo contexto de actualización del modelo de desarrollo del país.
\end{abstract}

Palabras clave: Política agraria; Desarrollo; Cooperativismo.

$1 \quad$ Licenciada en Sociología. Profesora Asistente del Departamento de Sociología. Facultad de Ciencias Sociales. Universidad Central "Marta Abreu” de Las Villas, Cuba. E-mail: liennygp@uclv.cu.

2 Doctor en Ciencias Económicas. Profesor Titular del Departamento de Marxismo. Dirección de Marxismo Leninismo e Historia. Universidad Central "Marta Abreu” de Las Villas, Cuba. E-mail: jaime@uclv.edu.cu.

3 Doctora en Economía. Profesora Titular de Economía Política y Cooperativismo. Facultad de Ciencias Económicas. Universidad Central “Marta Abreu” de Las Villas, Cuba. E-mail: grizel@uclv.edu.cu. 
Resumo: O cooperativismo em Cuba viu-se potencializado a partir do triunfo da Revolução do 1 de janeiro de 1959. Apesar disso, o país adotou a via da estatização como via de socialização socialista. Apostou na unidade e na cooperação do movimento campesino cubano para a produção e a prestação de serviços. Portanto, o problema agrário sempre fez parte da estratégia de desenvolvimento de Cuba. Os anos abrangidos pelo cooperativismo agrícola e a extensão do cooperativismo a novas esferas de produção e serviços complicam o cenário atual e colocam novos desafios. Isto traz à luz a necessidade de olhar para o futuro do cooperativismo em Cuba e para as políticas que favoreceram ou dificultem seu desenvolvimento, sem se esquecer da importância da participação dos sujeitos na formulação das mesmas. Por essa razão, este trabalho tem como objetivo caracterizar as ações do Estado cubano no fortalecimento do cooperativismo e identificar seus principais desafios nas Políticas Públicas. Isso foi possível com base em ampla revisão bibliográfica e análise de documentos (leis, decretos) que permitem validar a análise bibliográfica realizada e identificar os desafios do cooperativismo no novo contexto de atualização do modelo de desenvolvimento do país.

Palavras chave: Política agraria; Desenvolvimento; Cooperativismo.

\begin{abstract}
Cooperativism in Cuba was strengthened after the triumph of the Revolution on January 1, 1959. Despite this, the country adopted the path of nationalization as a means of socialist socialization. It relied on the unity and cooperation of the Cuban peasant movement for the production and provision of services. Therefore, the agrarian problem was always part of Cuba's development strategy. The years covered by agricultural cooperativism and the extension of cooperativism to new spheres of production and services complicate the current scenario and pose new challenges. This raises a need to look at the future of cooperativism in Cuba and the policies that have favored or hindered its development; without forgetting the importance of the participation of the subjects in this formulation. This work therefore characterizes the actions of the Cuban State in strengthening cooperativism, and identifies its main challenges in terms of Public Policies. This was supported by a broad bibliographic review and an analysis of documents (laws, decrees) that enabled us to validate the bibliographic analysis carried out and identify the challenges facing cooperativism in the new context of modernization of the country's development model.
\end{abstract}

Keywords: Agrarian policy; Development; Cooperativism.

\title{
INTRODUCCIÓN
}

La cooperativización en Cuba ha transitado por diferentes etapas, vinculadas con el proceso de reorganización social, primeramente, a partir de los cambios impulsados por el gobierno revolucionario y consecutivamente los promovidos por la opción socialista. Aunque desde la Constitución del 1940 ya se fomentaba la idea de la cooperativización, no fue hasta 1982 que se aprobó la Ley de Cooperativas Agrarias (Piñeiro Harnecker, 2014). 
La cooperativización en la agricultura fue reflejo del carácter de los primeros cambios revolucionarios (popular, agrario y antimperialista) y en armonía con la estrategia cubana de desarrollo ${ }^{4}$ que tuvo como pivote principal a la industria azucarera. Por tanto, las particularidades del cooperativismo en Cuba respecto a otros países son producto de "(...) la estructura agraria cubana; la creación mayorista, en los primeros momentos de las Cooperativas de Crédito y Servicios (CCS); la diversidad de formas de cooperación del trabajo; la relativa lentitud del proceso de cooperación productiva (Autores, 2002)".

Por tanto, las etapas del desarrollo del cooperativismo en Cuba están asociadas a los intereses de desarrollo del país expresados en la estrategia de desarrollo emprendida por el gobierno revolucionario.

La reforma de los 90 del sigloXXen Cuba posibilitó "cuotas de descentralización a favor de los decisores empresariales y de los gobiernos locales" (Espina, 2006), estableciendo estructuras que ayudan a contrarrestar la crisis y establecer estrategias locales de desarrollo. Desde el punto de vista del cooperativismo y su papel en la estrategia de desarrollo del país, comienzan a buscarse soluciones ante la crisis agraria y económica y social en general. Vuelve a la palestra la necesidad de la cooperativización como forma de socialización socialista, impulsándose la formación de cooperativas de trabajadores, denominadas Unidades Básicas de Producción Cooperativa (UBPC). Se desplegó un proceso de consolidación y perfeccionamiento de las cooperativas creadas (CPA y CCS, respectivamente) como resultado del V Congreso del PCC en 1997. Todos estos cambios son continuación del proceso de cooperativización iniciado y resultado de una serie de contradicciones que manifestó la sociedad cubana y en especial sus localidades, lo que propició a que se comenzara a valorizar el ámbito local (Guzón Camporredondo, 2006).

En los lineamientos para la Política Económica y Social del Partido y la Revolución, aprobados en $6^{\text {to. }}$ Congreso del Partido Comunista de Cuba en el año 2012, se resaltó la necesidad de ampliar el sector cooperativo, fomentando la creación de cooperativas con diferentes objetos sociales, en particular

$4 \quad$ La estrategia cubana de desarrollo socioeconómico tuvo y tiene hoy un objetivo fundamental: lograr la independencia nacional, la justicia social y la equidad, donde los seres humanos son los protagonistas y beneficiarios directos del desarrollo. 
las cooperativas no agropecuarias, (producción, prestación de servicios y comercialización); como continuidad y ampliación del sector cooperativo en el país.

Sin embargo, se reconoce la inexistencia, a nivel social y en las estructuras de dirección, de una cultura cooperativa, formas de pensar y actuar que favorezcan el movimiento a pesar de la práctica del cooperativismo agropecuario. Sobresalen incongruencias en el proceso de socialización, las formas y vías de asociatividad, sobre todo en el no reconocimiento de espacios de cooperación inter cooperativas y entre los tipos de economías y hereda una serie de restricciones (Donéstevez Sánchez, 2011) sufridas por las cooperativas agropecuarias a partir de decisiones tomadas centralmente o desde las empresas de las diferentes ramas agropecuarias. Las experiencias del sector cooperativo agropecuario deben contribuir al proceso de implementación y formación de las nuevas cooperativas, posibilitando una visión de la evolución del sector cooperativo que lo integre en un solo movimiento, sin considerarlos como departamentos estancos (agropecuario y no agropecuario).

Diversas son las perspectivas teóricas que han abordado el cooperativismo como factor dinamizador del desarrollo dentro y fuera de Cuba.

En su generalidad se expresa que la propia esencia del cooperativismo (a partir de sus valores y principios) y su ubicación en una localidad determinada, hace del sector un actor del desarrollo de las localidades. Entre sus potencialidades para constituirse en palanca del desarrollo se resaltan las siguientes: El cooperativismo como fin en sí mismo. Primero, por la democracia en las organizaciones empresariales basadas en la participación en los procesos de producción y distribución. Segundo, por la intercooperación, mecanismo para hacer trascender y para propagar la democracia (García -Gurtiérrez Fernández, 1999).

Desde los principios y desde sus características organizacionales las cooperativas se identifican con el desarrollo local. ${ }^{5}$ El fortalecimiento empresarial y de sus asociados como objetivo tradicional del cooperativismo. Expresado en la gestión cooperativa. ${ }^{6}$ El cooperativismo como actividad directamente ligada

$5 \quad$ Ver COQUE MARTÍNEZ, J. 1999. Industrialización en el entorno local a través de (sociedades) cooperativas. Revista de Estudios Cooperativos (REVESCO), 68. Ver FERNÁNDEZ, G., NARVÁEZ, M. \& SENIOR, A. 2010. Organizaciones cooperativas en el contexto del desarrollo local: una aproximación para su estudio. Revista Venezolana de Gerencia (RVG), 49.

6 Ver STRYJAN, Y. 1999. Cooperativas, emprendimientos colectivos y desarrollo local. Publicación del Centro de 
a la comunidad desde sus fines como desde su composición. ${ }^{7}$ Las cooperativas, son capaces de escapar al límite de la pequeña escala de la producción y los servicios, mediante los lazos de cooperación que establecen entre el pequeño productor campesino y artesano. ${ }^{8}$ Constituyen una vía para insertarse en mejores condiciones en el mercado, para aumentar los niveles de ingresos de los socios y posibilitarle acceso a beneficios sociales así como formalizar la situación (desempleo o ilegalidad) de los socios desde el punto de vista laboral. ${ }^{9}$

Aunque se reconocen las potencialidades del cooperativismo en su contribución al desarrollo local, también se identifican algunas limitaciones o retos para poder cumplir cabalmente su papel en ese sentido. Entre las limitaciones se sugieren: el bajo nivel de capacitación en diferentes temáticas, sobre todo las relacionadas con los principios cooperativos (Jiménez Guethón, diciembre 2008); la débil articulación entre la cooperación y la comunidad (Barbosa and Grippo, 2000); la inobservancia de los principios y valores del cooperativismo, lo que produce una metamorfosis en su estructura y funciones (Maldovan Bonelli, 2012); la insuficiente conexión de las cooperativas con el sistema público producto a las limitaciones entre cooperativas y Estado (Maldovan Bonelli, 2012).

Lo anteriormente expuesto permite visualizar al cooperativismo como un espacio con significativas capacidades para activar procesos de participación social, una vía específica de socialización socialista con potencialidades para favorecer el desarrollo socioeconómico local y un sector donde se propician lazos de cooperación en el trabajo y colectivización de los medios de producción. El universo cooperativo está determinado por el sistema de relaciones sociales de producción prevalecientes en la sociedad de que se trate. Es un motor impulsor del desarrollo y especialmente en las localidades cubanas donde el gobierno tiene la posibilidad de fomentar la cooperación entre los diferentes sectores y actores. Sin embargo, se carece de un análisis multidimensional de las potencialidades

Estudios de Estudios de Sociología del Trabajo. Universidad de Buenos Aires, Facultad de Ciencias Económicas. Instituto de Investigaciones Administrativas. Ver CRUZ REYES, J. \& PIÑEIRO HARNECKER, C. 2012. ¿Qué es una cooperativa? In: PIÑEIRO HARNECKER, C. (ed.) Cooperativas y socialismo. Una mirada desde Cuba. La Habana: Editorial Caminos.

7 Ver CUÑAT GIMENEZ, R. J. \& COLL SERRANO, V. 2007. ¿Contribuyen las cooperativas de reciente creación al desarrollo local? Una visión desde los principios cooperativos. Revista Venezolana de Economía Social, 13.

8 Ver DONÉSTEVEZ SÁNCHEZ, G. 2016. Cooperativismo y la cultura cooperativa en la transición socialista. In: KAIRÓS (ed.) Direito á CIUDADE e ao trabalho. Olhares de Brasil e Cuba. Curitiva, Brasil.

9 Ver MALDOVAN BONELLI, J. 2012. Trabajo, asociatividad y acción colectiva: el caso de las cooperativas de recuperadores urbanos. Trabajo y Sociedad, 18. 
y limitaciones que presenta el sector cooperativo configurado bajo la actual reforma, frente a las demandas impuestas por el desarrollo a escala local.

Los estudios sobre el cooperativismo en el país se han concentrado fundamentalmente en el medio agrario por constituir la forma históricamente adoptada. Por el contrario, el carácter emergente del asociativismo en otras ramas de la economía marca inexorablemente la naturaleza exploratoria de las indagaciones sociales emprendidas en este campo. Hasta el momento, entre los objetos de análisis realizados figura el proceso de implementación y formación de cooperativas no agropecuarias sobre la base de criterios, tales como: la motivación a partir de la nueva forma de organización del trabajo y la distribución de los ingresos y el ambiente laboral (Piñeiro Harnecker, 2015).

El nuevo escenario del cooperativismo en Cuba requiere no solo de la acción de Políticas Sociales, las cuales ha sido en su mayoría verticales y centralizadas. Sino que, para su correcto desenvolvimiento como forma productiva y como movimiento social requiere de la implementación de Políticas Públicas, donde "la deliberación y la discusión son partes intrínsecas (Arellano Gault, 2014)" del proceso.

Aunque las políticas agrarias llevadas a cabo por el país, desde el triunfo de la Revolución Cubana han influido en el quehacer de las cooperativas. El reto para Cuba está en proponer Políticas Públicas hacia el sector, para lo cual es indispensable la presencia en el contexto cubano de una Ley de Cooperativas. Pues la Historia del cooperativismo cubano en este sentido, refleja la ausencia de políticas enfocadas al cooperativismo en general, a pesar de los intentos hechos en las cooperativas agropecuarias.

El presente trabajo pretende caracterizar las acciones del Estado cubano en la potenciación del cooperativismo e identificar sus principales retos en materia de Políticas Públicas.

1. Política agraria y estrategia de desarrollo cubanas. Punto de partida, continuidad y potenciación del cooperativismo.

El proceso de cooperativización en Cuba fue potenciado primeramente en la agricultura. Sin embargo, las políticas emprendidas en el país no fueron dirigidas al sector cooperativo directamente. Sino que la política agraria cubana 
fue reflejo del carácter de los primeros cambios revolucionarios (popular, agrario y antimperialista) y en armonía con la estrategia cubana de desarrollo ${ }^{10}$ que tuvo como pivote principal a la industria azucarera. Por tanto, las etapas del desarrollo del cooperativismo en Cuba están asociadas con la estrategia de desarrollo emprendida por el gobierno revolucionario. De tal manera en el presente acápite se intentará visualizar las políticas que han favorecido el proceso de cooperativización en su relación y congruencias con la estrategia de desarrollo cubana en sus diferentes etapas.

Con el triunfo del gobierno revolucionario en los primeros años de la revolución como antecedente a la primera etapa de la estrategia de desarrollo fueron creadas las primeras cooperativas agrícolas, resultado de la expropiación de las grandes fincas dedicadas fundamentalmente al cultivo de la caña de azúcar, arroz, etc. Esto se convirtió poco después en empresas cooperativas- estatales, resultado de la combinación de los principios estatales y cooperativos.

La Primera Ley de Reforma Agraria trajo como resultado las Asociaciones Campesinas, "lo que llevó a la creación de las Cooperativas Integrales de Producción (Hereira Domínguez, 2015)". La primera ley en su Capítulo V, artículo 43 abogaba por que el Instituto Nacional de Reforma Agraria (INRA), fomentara cooperativas agrícolas, pero con un administrador designado por el Instituto velando por el buen desenvolvimiento de la nueva organización económica. Desde sus inicios las políticas que favorecía el cooperativismo en el país adolecieron de una excesiva centralización que de alguna manera afectiva su autonomía como empresa.

Además de las Asociaciones Campesinas ${ }^{11}$, en estos primeros años predominaron como formas de organización económica y civil las Cooperativas de Créditos y Servicios y las Sociedades agropecuarias. Estas formas de organización fueron acompañadas por administraciones generales creadas en entre los años 1961-1962. Las administraciones fueron las siguientes: Administración General de Granjas del Pueblo, Administración General de Cooperativas

10 La estrategia cubana de desarrollo socioeconómico tuvo y tiene hoy un objetivo fundamental: lograr la independencia nacional, la justicia social y la equidad, donde los seres humanos son los protagonistas y beneficiarios directos del desarrollo.

11 Constituían las asociaciones de base de la Asociación Nacional de Agricultores Pequeños, creada en 1961. Para el año 1962 ya el país contaba con 2339 asociaciones campesinas distribuidas en las provincias: Pinar del Río, La Habana, Matanzas, Las Villas, Camagüey y Oriente. (Ver VALDÉS PAZ, J. 2009. Procesos de organización agrarias en Cuba. 1959-2006, La Habana, Fundación Antonio Núñez Jimenez de la naturaleza y el hombre. 
Cañeras y Administración General de Fincas. Estas formas generaron una serie de contradicciones, por su carácter general y la homogeneidad en los aparatos administrativos, no teniendo en cuenta las diferencias entre cada una de las regiones (Valdés Paz, 2009).

Existen conceso en que la primera de las etapas de la estrategia de desarrollo del país abarca de 1962 a 1964 y se caracterizó por tener una estrategia basada en la industrialización acelerada a partir de la expansión de la industria pesada, la diversificación de la agricultura, la redistribución del fondo agrícola cañero y sustitución creciente de las importaciones por la producción nacional. En el proceso de diversificación de la agricultura se vio la impronta del cooperativismo. Surgen cooperativas con diversos objetos sociales díganse: cooperativas agrícolas, pecuarias, agropecuarias, avícolas, carboneras, madereras, henequeneras, paperas, cañeras, y pesqueras (Valdés Paz, 2009). La Segunda Ley de Reforma Agraria en 1963, eliminó las relaciones capitalistas de producción y afectó directamente al sector privado.

Octubre de 1960 marca el paso de la etapa democrático, popular, agrario y antimperialista a la etapa socialista. Pues se había liquidado el sector capitalista. A partir de aquí comienza el período de transición al socialismo en Cuba, no sólo por la declaración política del Comandante en Jefe Fidel Casto en 1961, sino también porque desde el punto de vista económico se había liquidado el gran capital nacional y extranjero en el país.

La segunda etapa abarca el período de 1964 a 1970 la estrategia estuvo caracterizada por un modelo Agroindustrial Exportador. La elaboración del plan tuvo un carácter extremadamente centralizado. Hacia 1967 concluye la dualidad de mecanismos y se comienza a aplicar un sistema de registro y control (Pérez Villanueva and Torres Pérez, 2013). En el período se mantiene la estructura organizativa en productores individuales, Cooperativas de Créditos y Servicios y Sociedades Agropecuarias impactando el carácter centralizado, planificado y de control en la agricultura y sus formas organizativas. Un ejemplo de esto son los Planes dirigidos (zonas de alta densidad campesina y diversidad en las producciones), los Planes especializados (unidad del sector estatal y las fincas 
campesinas con un plan común) y los Planes integrales (se organizan y funcionan como empresas estatales agropecuarias) (Valdés Paz, 2009).

La tercera etapa se desarrolla de 1975 a 1985 donde la estrategia estuvo caracterizada por un Modelo de Desarrollo Industrial con una industrialización acelerada. Se comienzan a elaborar los planes quinquenales. El mercado es regulado y centralizado y en 1984 se crea el mercado Libre Campesino y el Mercado Paralelo. El modelo de gestión de este período estuvo basado en la implementación del Sistema de Dirección y Planificación de la Economía. Se despliega la planificación centralizada basada en los balances materiales y un sistema único de distribución de los factores de la producción y de los resultados (Pérez Villanueva and Torres Pérez, 2013).

Los impactos del Primer Congreso del Partido Comunista de Cuba (1975) y el Segundo Congreso (1980) impactaron en este período, sobre todo en la aceleración del modelo socialista ${ }^{12}$, resaltando la vía cooperativización como una vía socialista, fundamentalmente en la agricultura cubana. El movimiento cooperativo se concentró en el auge de las Cooperativas de Producción Agropecuaria $^{13}$ (CPA) cuya base está en la propiedad común sobre la tierra y demás medios de producción. Estas se caracterizaban por un fuerte apoyo estatal y su proceso de conformación comenzó a finales de 1976 y a finales de 1979 se habían constituido más de 800 CPA con 26454 socios aproximadamente con un promedio de 33 socios por cooperativa (Valdés Paz, 2009).

Ya desde 1976 en la Constitución de la República de Cuba en sus artículos 19 y 20 se reconoce la propiedad cooperativa en el sector agropecuario y el derecho de los agricultores pequeños de asociarse. Para 1977 en la Resolución del V Congreso de la ANAP, celebrado el 17 de mayo de ese año, se resalta la importancia de la cooperativización y el fomento de otras formas colectivas como las Sociedades Agropecuarias, las brigadas de macheteros campesinos y de ayuda mutua, así como las organizaciones de mujeres campesinas en el seno

12 En la tesis resolución del Primer Congreso del Partido Comunista de Cuba, se reconoce que luego de la Segunda ley de Reforma Agraria el sector socialista de la agricultura (Granjas estatales y cooperativas cañeras) abarcaban en $70 \%$ de la superficie del país. Ver (PCC), P. C. D. C. 1984. Tesis y Resolución del Primer Congreso del Partido Comunista de Cuba de 1976. In: NAVARRETE ACEVEDO, C. (ed.) Legislación y documentos sobre derecho agrario cubano. La Habana: Universidad de La Habana. Facultad de Derecho. Ministerio de Educación Superior.

13 Consideradas como formas superiores de organización de la producción de la agricultura socialista, junto a las Empresas Agropecuarias Estatales. 
de las Brigadas FMC-ANAP ((PCC), 2011). Se reconoce además las posibilidades de las Sociedades Cooperativas y las Cooperativas de Créditos y Servicios de convertirse o transitar a formas superiores de producción.

De alguna manera como consolidación de este proceso en julio de 1982 se establece la Ley No. 36 de Cooperativas Agropecuarias. La misma recoge las dos formas cooperativas fundamentalmente desarrolladas en el país: las CPA y las CCS. Declarándose en el documento sus principios y fines, reconociendo su autonomía respecto al estado y su personalidad jurídica.

La creación del mercado Libre Campesino, al que podían acceder las CPA, significó un fortalecimiento de estas formas cooperativas, sin embargo estos cambios asociados al resquebrajamiento de los valores y principio cooperativos en estas formas trajo una serie de consecuencias: "carrera desenfrenada por las ganancias sin preocupación por la eficiencia productiva; atención priorizada a los valores en detrimento de los valores de uso; absolutización de los mecanismos económicos sin tener en cuenta la formación integral del cooperativista; subestimación de las producciones fundamentales (caña, tabaco, etc.) donde tenían un gran peso; desvinculación de los ingresos respecto a los resultados del trabajo y otras (Alemán and Figueroa Albelo, 2005)".

El cuarto período recorre de 1986 a 1989 caracterizado por una recesión económica: rectificación de errores y tendencias negativas. Se detiene el proceso de implementación del Sistema de Dirección y Planificación de la Economía y en 1986 se le pone fin al Mercado Libre Campesino (Pérez Villanueva and Torres Pérez, 2013). En este periodo se vio frenado el proceso de cooperativización producto al agotamiento del modelo agrícola cubano, disminuyendo la superficie cooperativizada. En varios espacios se discutieron las posibilidades de superar las deficiencias: II Encuentro de Cooperativas, VII Congreso de la ANAP, III Congreso del PCC.

En este último documento resume la situación del contexto de la siguiente manera: "El desarrollo de las cooperativas de producción agropecuaria impondrá la necesidad de perfeccionar esta forma socialista de producción. Por eso se deberán aplicar medidas que eleven sustancialmente la organización de la producción y 
el trabajo en estas empresas así como sus relaciones con el sector estatal de la economía... Así mismo se instrumentarán mecanismos económico-financieros que estimulen la incorporación de los campesinos privados a formas superiores de producción ((PCC), 1986)".

De 1990 a 2010 se despliega la quinta etapa donde la estrategia estuvo dirigida a la reforma y ajuste económico producto de la crisis económica. Se deja a un lado la planificación quinquenal y a largo plazo. Aparecen también diferentes programas, entre ellos: Alimentario, Energético, de piezas de repuestos y tecnológicos de avanzada, de exportaciones tradicionales, de exportaciones no tradicionales, de exportaciones de servicios, etc. Se le da un mayor espacio al mercado con el Mercado Libre Agropecuario, el Mercado de Artículos Industriales y Artesanales, el Mercado de Divisas y el Mercado de Precios Topados o Mercado Agropecuario Estatal. El modelo de gestión se caracterizó por la planificación centralizada basada más en balances financieros. Se comienza a delimitar las funciones estatales de los empresariales, acompañados de un proceso de Perfeccionamiento Empresarial ((PCC), 1986).

En la etapa comienzan a buscarse soluciones ante la crisis agraria y económica y social en general. Por lo que se vuelve a considerar la necesidad de la vía de la cooperativización como forma de socialización socialista, impulsándose la formación de cooperativas de trabajadores, entre 1993-1994, denominadas Unidades Básicas de Producción Cooperativa (UBPC). Este proceso tuvo sus contradicciones.

En el V Congreso del PCC se planteaba que la creación de estas unidades "modificó las condiciones de trabajo e ingresos de los obreros de las granjas estatales hacia cooperativas, recibiendo las tierras en usufructo gratuito e ilimitado en el tiempo y los demás medios necesarios en condiciones favorables de créditos, además de excepciones fiscales y subsidios presupuestarios cuando se ha querido ((PCC), 2011). Sin embargo, no es menos cierto que las UBPC heredaron problemas económicos- financieros de las antiguas formas de gestión, además de incongruencias en el proceso de creación. 
Se desplegó un proceso de consolidación de las CPA y de fortalecimiento de las CCS como resultado también del V Congreso del PCC ${ }^{14}$ en 1997. En el 2008 el movimiento cooperativo se robusteció con la puesta en marcha del Decreto Ley No. 259 que permitió la entrega de tierras en usufructo a personas jurídicas y naturales.

La sexta etapa abarca de 2010 hasta la actualidad cuya estrategia está caracterizada por la actualización del modelo cubano: modelo agroindustrial exportador y de sustitución de importaciones y fortalecimiento del Sector Servicios, sobre todo los relacionados con la producción de conocimientos. Se retoma la planificación quinquenal y estratégica y se les da mayor espacio a los mercados de libre formación de precios bajo regulación. Como modelo de gestión se tiene en cuenta el balance plan-mercado. El sector cooperativo manifestó una aplicación al sector no agropecuario.

En los Lineamientos de la Política económica y social del Partido y la Revolución aprobados en el VI Congreso del Partido Comunista de Cuba en el año 2011; se resalta la necesidad de ampliar el sector cooperativo. Se fomenta la creación de cooperativas de 1er grado (lineamiento 25 ) y se reconocen las cooperativas de 2do grado (lineamiento 29) a partir de la creación de las primeras ambas con objetos sociales diversos (producción, prestación de servicios y comercialización).

De tal manera la estrategia de desarrollo cubana contempla a las cooperativas como una forma de propiedad socialista ${ }^{15}$. Por lo que el Estado cubano propone un marco regulatorio (La O Serra, 2014) para el funcionamiento de las cooperativas en el sector no agropecuario resumido a continuación:

El Decreto-Ley No. 305 de 11 de diciembre de 2012. Que establece con carácter experimental las normas de constitución, funcionamiento y extinción de las cooperativas no agropecuarias.

El Decreto-Ley No. 306 de 11 de diciembre de 2012. Establece el Régimen Especial de Seguridad Social.

14 Se plantea la necesidad de que las CPA continuarán su sistema de gestión económico productivo a partir de "la aplicación concreta de nuevos conceptos de organización, la introducción de la ciencia y la técnica y el fortalecimiento de sus aparatos contables para garantizar la elevación de sus producciones y eficiencia". Ver (PCC), P. C. D. C. 2011. Resolución económica del V Congreso del Partido Comunista de Cuba de 1997

15 Ver Lineamiento 159. En (PCC), P. C. D. C. 2016. Plan Nacional de desarrollo económico y social hasta el 2030 : propuesta de versión de la nación, ejes y sectores estratégicos. 
El Decreto No. 309 de 11 de diciembre de 2012. Sobre el Reglamento del Decreto-Ley No. 305/2012.

La Ley No. 113/2012. Del Sistema Tributario, Título II Del Impuesto sobre las Utilidades, Capítulo II De los Regímenes Especiales, Sección IV Del Régimen Especial para el Sector Cooperativo no Agropecuario.

La Resolución No. 570/2012, del Ministerio de Economía y Planificación. Aprueba y pone en vigor el procedimiento de licitación.

La Resolución No. 427/2012, del Ministerio de Finanzas y Precios. Establece el tratamiento de precios y de patrimonio estatal, la constitución de un fondo público de fideicomiso y la Norma Específica de Contabilidad para las cooperativas no agropecuarias.

La naturaleza de estas cooperativas (1er grado) ha sido heterogénea, proceden de personas naturales (trabajadores por cuenta propia, productores individuales) o personas jurídicas (empresa estatal), pero de manera general expresan las intenciones del país de ampliar el cooperativismo más allá del sector agropecuario.

En el VII Congreso del Partido Comunista de Cuba en 2016, se reafirma la idea del cooperativismo, sobre todo en el sector no agropecuario. Sin embargo, la propuesta de estrategia de desarrollo enfoca el cooperativismo en general. Así es que se refleja en la posibilidad de la creación de cooperativas en diferentes sectores y actividades. En el lineamiento 161, se reconoce a las cooperativas como "una forma de propiedad colectiva en la que sus trabajadores permanentes son socios con iguales derechos, que participan de la distribución de utilidades de acuerdo con el trabajo aportado" (PCC), 2016).

De manera general se puede decir que en el último período la concepción, elaboración y aprobación de la estrategia de desarrollo del país ha desplegado un nivel de participación y consulta. Podría decirse, muy intrépidamente que se están dando pasos para la elaboración de políticas de carácter público. Además, existe una congruencia entre la estrategia de desarrollo del país y las políticas enfocadas al sector cooperativo. 
La práctica del cooperativismo cubano, necesita de la superación de las siguientes contradicciones (Muñoz Alfonso et al., 2011):

Contradicción entre el ser y el deber ser de dichas organizaciones.

Contradicción entre la escasez de recursos materiales y financieros y las necesidades de su desarrollo.

Contradicción entre la ayuda que reciben y la necesidad de la autonomía.

Contradicción entre la rentabilidad económica y financiera de la cooperativa y la de los socios.

Superación de la rigidez que presenta la legislación agraria referente a la formación de cooperativas y la limitada concepción que se tiene respecto a las gradaciones que puedan presentar la cooperación en el sector.

Dada la multiplicidad de normas es necesario un proceso de educación sobre el sistema jurídico que ampara el cooperativismo en el país, resaltando la jerarquía de las normas.

Fomentar un sistema de gestión para las nuevas cooperativas (cooperativas no agropecuarias), basado en la experiencia acumulada por las cooperativas agrícolas que esté acorde a las condiciones actuales de la economía cubana (Donéstevez Sánchez et al., 2011).

Potenciar un modelo de organización de las cooperativas, asentado en sistemas democráticos y flexibles que faciliten procesos participativos y la especialización productiva atendiendo a particularidades de cada sector y niveles alcanzados por la socialización del trabajo (Donéstevez Sánchez et al., 2011).

\section{REFERENCIAS DE LAS FUENTES CITADAS}

(PCC), P. C. D. C. 1984. Tesis y Resolución del Primer Congreso del Partido Comunista de Cuba de 1976. In: NAVARRETE ACEVEDO, C. (ed.) Legislación y documentos sobre derecho agrario cubano. La Habana: Universidad de La Habana. Facultad de Derecho. Ministerio de Educación Superior.

(PCC), P. C. D. C. 1986. Resoluciones aprobadas por el III Congreso del Partido Comunista de Cuba. Tesis y Resoluciones sobre el perfeccionamiento del sistema de dirección y planificación del al economía. La Habana: Editora Política.

(PCC), P. C. D. C. 2011. Resolución económica del V Congreso del Partido Comunista de Cuba de 1997. 
(PCC), P. C. D. C. 2016. Plan Nacional de desarrollo económico y social hasta el 2030: propuesta de versión de la nación, ejes y sectores estratégicos.

ALEMÁN, S. \& FIGUEROA ALBELO, V. 2005. El modelo cooperativo campesino en Cuba, La Habana.

ARELLANO GAULT, D. 2014. Introducción: la enseñanza de la evaluación de políticas públicas. In: RÍOS CÁZARES, A. (ed.) La evaluación de políticas públicas en América Latina: métodos y propuestas docentes. México: Centro CLEAR para América Latina-Red Inter-Americana de Educación en Administración Pública. Centro de Investigación y Docencia Económicas A.C.

AUTORES, C. D. 2002. Economía política de la construcción del socialismo. Fundamentos generales, La Habana.

BARBOSA, O. \& GRIPPO, R. 2000. Cooperativismo, globalización y desarrollo local. CIESER, Encuentro Río Cooperativo.

COQUE MARTÍNEZ, J. 1999. Industrialización en el entorno local a través de (sociedades) cooperativas. Revista de Estudios Cooperativos (REVESCO), 68.

CRUZ REYES, J. \& PIÑEIRO HARNECKER, C. 2012. ¿Qué es una cooperativa? In: PIÑEIRO HARNECKER, C. (ed.) Cooperativas y socialismo. Una mirada desde Cuba. La Habana: Editorial Caminos.

CUÑAT GIMENEZ, R. J. \& COLL SERRANO, V. 2007. ¿Contribuyen las cooperativas de reciente creación al desarrollo local? Una visión desde los principios cooperativos. Revista Venezolana de Economía Social, 13.

DONÉSTEVEZ SÁNCHEZ, G. 2016. Cooperativismo y la cultura cooperativa en la trnasición socialista. In: KAIRÓS (ed.) Direito á CIUDADE e ao trabalho. Olhares de Brasil e Cuba. Curitiva, Brasil.

DONÉSTEVEZ SÁNCHEZ, G., LÓPEZ DE ARMAS, G. \& GARCÍA RUIZ, J. 2011. El nuevo cooperativismo en Cuba del siglo XXI. Estúdios de Direito Cooperativo e Cidadania, 4.

DONÉSTEVEZ SÁNCHEZ, G. G. R., JAIME; FAJARDO NÁPOLES, LUISA; FIGUERAS MATOS, DAGOBERTO; MUÑOZ ALFONSO, YISEL; MARTIRENA HERNÁNDEZ, JOSÉ F. 2011. El cooperativismo del siglo XXI. Estudos de Direito Cooperativo e Cidadania, 4.

ESPINA, M. 2006. Apuntes sobre el concepto de desarrollo y su dimensión territorial. In: ACADEMIA, E. (ed.) Desarrollo Local en Cuba. La Habana (Cuba).

FERNÁNDEZ, G., NARVÁEZ, M. \& SENIOR, A. 2010. Organizaciones cooperativas en el contexto del desarrollo local: una aproximación para su estudio. Revista Venezolana de Gerencia (RVG), 49.

GARCÍA -GURTIÉRREZ FERNÁNDEZ, C. 1999. Cooperativismo y Desarrollo Local. Revista de Estudios Cooperativos (REVESCO), 68. 
Novos Estudos Jurídicos

GUZÓN CAMPORREDONDO, A. 2006. Desarrollo Local en Cuba, La Habana, Editorial Academia. HEREIRA DOMÍNGUEZ, M. 2015. El cooperativismo y su importancia para el desarrollo del municipio de Manicaragua. Universidad Central "Marta Abreu" de Las Villas (UCLV).

JIMÉNEZ GUETHÓN, R. M. diciembre 2008. Cooperativas agrícolas en Cuba y su relación con el desarrollo local. Univeristas Forum, Vol. 1, 6.

LA O SERRA, B. 2014. Decreto - Ley No. 305 "De las Cooperativas no agropecuarias". In: COLECTIVOS, O. N. D. B. (ed.). La Habana.

MALDOVAN BONELLI, J. 2012. Trabajo, asociatividad y acción colectiva: el caso de las cooperativas de recuperadores urbanos. Trabajo y Sociedad, 18.

MUÑOZ ALFONSO, Y., LÓPEZ GONZÁLES, I., CAMPOS PÉREZ, Y. \& EMBALÓ QUIJANO, Y. 2011. Dimensión jurídica del cooperativismo en Cuba ante las nuevas exigencias económicas y sociales del país. Estúdios de Direito Cooperativo e Cidadania, 4.

PÉREZ VILLANUEVA, O. \& TORRES PÉREZ, R. 2013. Economía Cubana, ensayos para una reestructuración necesaria.

PIÑEIRO HARNECKER, C. 2014. Las cooperativas en Cuba: situación actual y perspectivas. In: A., M. \& GONZÁLEZ-CORZO, M. (eds.) Reformando el Modelo Económico Cubano. Nueva York: Bildner Center for Western Hemisphere Studies.

PIÑEIRO HARNECKER, C. 2015. Nuevas Cooperativas cubanas: logros y dificultades. In: PÉREZ VILLANUEVA, O. E. \& TORRES PÉREZ, R. (eds.) Miradas a la Economía Cubana. Análisis del sector no estatal. La Habana: Editorial Caminos.

STRYJAN, Y. 1999. Cooperativas, emprendimientos colectivos y desarrollo local. Publicación del Centro de Estudios de Estudios de Sociología del Trabajo. Universidad de Buenos Aires, Facultad de Ciencias Económicas. Instituto de Investigaciones Administrativas.

VALDÉS PAZ, J. 2009. Procesos de organización agrarias en Cuba. 1959-2006, La Habana, Fundación Antonio Núñez Jimenez de la naturaleza y el hombre. 